\title{
MEASURES AND TENSORS
}

\author{
BY \\ JESUS GIL DE LAMADRID $\left(^{1}\right)$
}

1. Introduction. The present work is divided into two parts. Part I, from which the title of the paper derives, has to do with the interpretation of tensor products of measure spaces as spaces of vector valued measures. Earlier results of the kind discussed here are due to Grothendieck [10], who showed in a much more general context that $L_{1}(\mu) \otimes_{\gamma} F=L_{1}(\mu, F)$, the corresponding space of Bochner integrable mappings, and that $l_{1} \otimes_{\lambda} F$ is the space of all unconditionally convergent series of vectors in $F$. Here $\mu$ is a Radon measure on a compact Hausdorff space $S, F$ is an arbitrary Banach space and $\gamma$ and $\lambda$ are, respectively, the greater cross norm and the least cross norm of Schatten [15]. The spaces $L_{1}(\mu)$ (hence $l_{1}$ ) can be considered as spaces of scalar measures and the corresponding vector valued mappings as vector measures. In the present article we replace $L_{1}(\mu)$ and $l_{1}$ by the dual $C^{\prime}(S)$ of $C(S)$. The space $C^{\prime}(S)$ is the space of all regular Borel scalar measures on $S$. The first problem is to determine the spaces of vector measures that correspond to the tensor products $C^{\prime}(S) \otimes_{\gamma} F$ and $C^{\prime}(S) \otimes_{\lambda} F$. This is done, in part (Theorems 4.1 and 4.2), with the help of the theory of vector measures of bounded variation and the theory of vector measures of bounded semivariation (weak measures in our nomenclature). It turns out that, under the resulting identification of tensors with measures, $\gamma$ corresponds to the total variation and $\lambda$ to the total semivariation.

Next we consider the duality of $C(S) \otimes_{\gamma} F$ and $C(S) \otimes_{\lambda} F$. As for determining the dual of $C(S) \otimes_{\lambda} F$, we have, because of the relation $C(S) \otimes_{\lambda} F$ $=C(S, F)$, that the question reduces to a known Riesz-type theorem [17]. As for determining the dual $\left[C(S) \otimes_{\gamma} F\right]^{\prime}$ of $C(S) \otimes_{\gamma} F$ we have (Schatten [15]), that this dual is $\mathscr{L}\left[C(S), F^{\prime}\right]$, the space of all bounded linear $T: C(S)$ $\rightarrow F^{\prime}$, and is identified below as a space of vector measures, by means of an extension of a theorem of Bartle, Dunford and Schwartz [2].

The rest of Part I is devoted to showing how one can apply the theory outlined above to extend certain results of Grothendieck [10] on the duality of the tensor products $E \otimes_{\gamma} F$ and $E \otimes_{\lambda} F$, where $E$ and $F$ are Banach spaces, and to obtain new proofs of two of his theorems.

Presented in part to the Society, November 23, 1962, January 24, 1963 and August 29, 1963; received by the editors July 2, 1962 and, in revised form, July 24, 1963.

(1) The author is an ONR Research Associate at Yale University. A portion of this research was supported by the National Science Foundation through the grant NSF-G-14137 at the University of Minnesota. 
Part II is devoted to the discussion of the following problem. An element of the tensor product $E \otimes_{\gamma} F$ defines, in a natural way, a bounded linear $U: E^{\prime} \rightarrow F$, called (Grothendieck) a nuclear transformation. Suppose that $F$ has an unconditional Schauder basis. The problem is to characterize all nuclear transformations $U: E^{\prime} \rightarrow F$, in terms of the basis. Our results fall somewhat short of this goal. Instead we give a concrete "basis expansion" for such nuclear transformations, which yields a necessary condition for a bounded linear transformation $U: E^{\prime} \rightarrow F$ to be nuclear. This condition can be applied easily in places where other existing criteria provide little information. We show that our characterization is not complete, in the sense that certain non-nuclear transformations can be represented in the given manner. In fact we show that a given Banach space $F$ has the property that the given basis expansion yields only nuclear transformations $U: E^{\prime} \rightarrow F$ (for arbitrary $E$ ) if and only if the basis of $F$ is absolute, i.e., $F$ is essentially $l_{1}$.

$\$ 2$ contains some essentially well-known background material on tensor products. In §3, we attempt to provide a similar background in the theory of vector valued measures. The main development of the present work begins in $\$ 4$. The term "scalar" may be interpreted throughout to mean either "real number" or "complex number." We do not specify either field of scalars because the distinction is immaterial for our considerations.

\section{Part I. Tensor Products of Functions and Measure Spaces}

2. Tensor products. In this section we outline those portions of the theory of topological tensor products developed by Schatten [15] and Grothendieck [10] which are relevant to our own work. Grothendieck presented his theory in the context of general locally convex topological vector spaces. We have endeavored here to interpret some of his material in the language of Banach spaces and in the spirit and notations of Schatten.

Let $E$ and $F$ be two Banach spaces. The symbol $\mathscr{L}(E, F)$ will denote the space of all bounded linear $T: E \rightarrow F$. A norm $\alpha$ on the algebraic tensor product $E \otimes F$ is said to be a cross norm if, for every $x \in E$ and $y \in F$, $\alpha(x \otimes y)=\|x\|\|y\| . E \otimes_{\alpha} F$ denotes the completion of $E \otimes F$ with respect to $\alpha$. Two specific cross norms are of particular interest. The "least cross norm" (a misnomer which we perpetuate) $\lambda$ is obtained through the natural algebraic imbedding of $E \otimes F$ into $\mathscr{L}\left(E^{\prime}, F\right)$. If under this imbedding $U^{i} \in \mathscr{L}\left(E^{\prime}, F\right)$ corresponds to a tensor

$$
t=\sum_{j=1}^{n} x_{j} \otimes y_{j}
$$

where $x_{j} \in E$ and $y_{j} \in F$, then, for every $x^{\prime} \in E^{\prime}$, 


$$
U^{t} x^{\prime}=\sum_{j=1}^{n}\left\langle x_{j}, x^{\prime}\right\rangle y_{j}
$$

We now define $\lambda(t)=\left\|U^{t}\right\| . \lambda$ is a cross norm. The greatest cross norm is defined by $\gamma(t)=\inf \sum_{j=1}^{n}\left\|x_{j}\right\|\left\|y_{j}\right\|$, where the inf is taken over all representations (2.1) of $t . \gamma$ is also a cross norm $\geqq \lambda$.

Soon we shall focus our interest on the spaces $E \otimes_{\gamma} F$ and $E \otimes_{\lambda} F$, but for the moment we consider any norm $\alpha$ on $E \otimes F$ such that

$$
\lambda \leqq \alpha \leqq \gamma
$$

It follows from (2.3) that $\alpha$ is a cross norm, for if $x \in E$ and $y \in F$, then $\|x\|\|y\|=\lambda(x \otimes y) \leqq \alpha(x \otimes y) \leqq \gamma(x \otimes y)=\|x\|\|y\|$. Every tensor $t \in E$ $\otimes_{\alpha} F$ has a representation

$$
t=\sum_{k=1}^{\infty} t_{k}
$$

where each $t_{k} \in E \otimes F$ and the series converges absolutely in $E \otimes_{\alpha} F$. This is established by means of the following standard argument. Since there exists a sequence $\overline{t_{1}}, \overline{t_{2}}, \cdots$, where each $\overline{t_{k}} \in \underline{E} \otimes F$, which converges to $t$ with respect to $\alpha$, we may assume that $\alpha\left(\bar{t}_{k+1}-\bar{t}_{k}\right)<1 / 2^{k}$ for every $k$, from which we get $t=\overline{t_{1}}+\sum_{k=1}^{\infty}\left(\overline{t_{k+1}}-\overline{t_{k}}\right)$, which is clearly of the form (2.4).

If $\alpha$ and $\beta$ are two norms on $E \otimes F$ such that $\gamma \geqq \alpha \geqq \beta \geqq \lambda$, there is a natural mapping $\Phi_{\alpha}^{\beta}: E \otimes_{\alpha} F \rightarrow E \otimes_{\beta} F$. If $t$ has a representation (2.4) in $E \otimes_{\alpha} \cdot F$, then $\Phi_{\alpha}^{\beta}(t)$ has exactly the same representation as an element of $E \otimes_{\beta} F$. From the definition of $\lambda$ it follows that there is an isometric imbedding represented by the inclusion $E \otimes_{\lambda} F \subset \mathscr{L}\left(E^{\prime}, F\right)$. From this and the discussion of $\Phi_{\alpha}^{\beta}$ it follows that for $\gamma \geqq \alpha \geqq \lambda$ one can assign to any $t \in E \otimes_{\alpha} F$ a linear transformation $U^{t} \in \mathscr{L}\left(E^{\prime}, F\right)$, given by (2.2) for $t \in E \otimes F$ and by the absolutely convergent series

$$
U^{t}=\sum_{k=1}^{\infty} U^{t_{k}}
$$

if $t$ has the representation (2.4) in $E \otimes_{\alpha} F$. $U^{t}$ is compact and continuous with respect to the weak* topology of $E^{\prime}$ and the weak topology of $F$. Also $\left\|U^{t}\right\|=\lambda\left[\Phi_{\alpha}^{\lambda}(t)\right] \leqq \alpha(t)$. In the case of a tensor $t \in E^{\prime} \otimes_{\alpha} F, U^{t}: E^{\prime \prime} \rightarrow F$, restricted to $E$ defines a bounded linear transformation $E \rightarrow F$ which we continue to designate by $U^{t}$. Clearly $U^{t}: E^{\prime \prime} \rightarrow F$ has the same transformation norm $\left\|U^{t}\right\|$ as $U^{t}: E \rightarrow F$.

The relation $\alpha \leqq \gamma$ makes it possible to define an algebraic imbedding represented by the inclusion

$$
\left(E \otimes_{\alpha} F\right)^{\prime} \subset{ }^{'} \mathscr{L}\left(E, F^{\prime}\right) .
$$


If $t_{T}^{\prime} \in\left(E \otimes_{\alpha} F\right)^{\prime}$ corresponds to $T \in \mathscr{L}\left(E, F^{\prime}\right)$ under this imbedding, we have, for every $t \in E \otimes F$,

$$
\left\langle t, t_{T}^{\prime}\right\rangle=\sum_{j=1}^{n}\left\langle y_{j}, T x_{j}\right\rangle
$$

if $t$ is represented by (2.1). For the general $t \in E \otimes_{\alpha} F$ represented by (2.4), we have $\left\langle t, t_{T}^{\prime}\right\rangle=\sum_{k=1}^{\infty}\left\langle t_{k}, t_{T}^{\prime}\right\rangle$. We have

$$
\alpha^{\prime}\left(t_{T}^{\prime}\right) \leqq\|T\|,
$$

where $\alpha^{\prime}$ is the dual norm of $\alpha$. We also have the algebraic imbedding symbolized by the inclusion $E^{\prime} \otimes F^{\prime} \subset \mathscr{L}\left(E, F^{\prime}\right)$, and represented by expressions similar to those for $U^{t}$ above. By virtue of the relation $\alpha \geqq \lambda$ we actually have the more refined imbedding represented by the inclusion

$$
E^{\prime} \otimes F^{\prime} \subset\left(E \otimes_{\alpha} F\right)^{\prime} \subset \mathscr{L}\left(E, F^{\prime}\right) .
$$

Consequently $\alpha^{\prime}$ is defined on $E^{\prime} \otimes F^{\prime}$ on which it is a cross norm.

For $\alpha=\gamma$ some of the above formulas become considerably simpler. In the place of (2.4) we have, for $t \in E \otimes F$,

$$
t=\sum_{j=1}^{\infty} x_{j} \otimes y_{j}
$$

where $x_{j} \in E, y_{j} \in F$ and the series converges absolutely in $E \otimes_{\gamma} F$. Also

$$
\gamma(t)=\inf \sum_{j=1}^{\infty}\left\|x_{j}\right\|\left\|y_{j}\right\|
$$

where the inf is taken over all representations (2.10) of $t$. The inclusion (2.6) becomes an identity:

$$
\left(E \otimes_{\gamma} F\right)^{\prime}=\mathscr{L}\left(E, F^{\prime}\right)
$$

which represents an isometry for the natural norms of those spaces.

A linear transformation $U: E^{\prime} \rightarrow F$ is said to be nuclear (Grothendieck) if it is of the form $U^{t}$, for $t \in E \otimes_{\gamma} F$.

More generally $U: E \rightarrow F$ is said to be nuclear if it is the restriction to $E \subset E^{\prime \prime}$ of a nuclear transformation $E^{\prime \prime} \rightarrow F$. No conflict arises, as can be easily proved, when one considers $E^{\prime} \subset E^{\prime \prime \prime}$. The assignment $t \rightarrow U^{t}$ (hence $\Phi_{\gamma}^{\lambda}$ ) need not be one-to-one, but it is one-to-one when restricted to the algebraic tensor product. For $t_{0} \in E \otimes F$ we define

$$
\tau\left(t_{0}\right)=\inf \gamma(t),
$$

where the inf is taken for all $t \in E \otimes_{\gamma} F$ such that $U^{t}=U^{t}$. It is not known whether $\tau$ and $\gamma$ are equal, but they may very well be different, since there 
may conceivably exist a tensor $t \in E \otimes_{\gamma} F^{\prime}$ not in $E \otimes F$ such that $U^{t}=U^{t_{0}}$ and $\gamma\left(t_{0}\right)>\gamma(t)$. At any rate we have $\gamma \geqq \tau \geqq \lambda$. Hence $\tau$ is a cross norm. We call it the trace norm and $E \otimes_{r} F$ the trace class, since they generalize concepts with the same names in the work of Schatten. It is not hard to see that $E \otimes_{\tau} F$ can be identified algebraically with the class of all nuclear transformations $U: E^{\prime} \rightarrow F$, that is, $\Phi_{\tau}^{\lambda}$ is one-to-one. Also $\Phi_{\gamma}^{\tau}$ is onto. For every $t \in E \cdot \otimes_{\tau} F$, we have also

$$
\tau(t)=\inf \sum_{j=1}^{\infty}\left\|x_{j}\right\|\left\|y_{j}\right\|,
$$

where the inf is taken for all representations (2.10) of $\bar{t} \in E \otimes_{\gamma} F$ such that $\Phi_{\gamma}^{r} \bar{t}=t$.

For tensor products of spaces of functions the following two representations are due to Grothendieck. The equations indicate isometries between the spaces involved with their usual norms.

$$
C(S, F)=C(S) \otimes_{\lambda} F
$$

[10, p. 89, Example 1].

$$
L_{1}(\mu, F)=L_{1}(\mu) \otimes_{\gamma} F
$$

$[10$, p. 59 , Theorem 2]. In $(2.15), C(S, F)$ is the space of all continuous $f: S \rightarrow F$ with the sup norm. In (2.16), $L_{1}(\mu, F)$ is the space of all Bochner $\mu$-integrable $f: S \rightarrow F$. Here, as well as in what follows, $S$ is assumed to be a compact Hausdorff space.

3. Vector measures. Let $S$ be a compact Hausdorff space, $\mathscr{B}(S)$ the Borel class of $S$ and $F$ an arbitrary Banach space. For any scalar Borel measure $\mu$ on $S$, we shall denote by $\bar{\mu}$ its variation measure. We now consider a mapping $m: \mathscr{B}(S) \rightarrow F^{\prime}$. For $y \in F$, we define a scalar valued set function $\mu_{y}$ by setting $\mu_{y}(A)=\langle y, m(A)\rangle$ for $A \in \mathscr{B}(S)$. We say that $m$ is a weak ${ }^{*}$ regular $F^{\prime}$-valued measure if for every $y \in F, \mu_{y}$ is a regular scalar valued measure. This concept is closely related to the concept of $F$-valued vector measure of Bartle, Dunford and Schwartz [2], which we term weak regular $F$-measure. In our setting a weak regular $F$-measure is simply a weak ${ }^{*}$ regular $F^{\prime \prime}$-measure with values in $F \subset F^{\prime \prime}$. We say that a weak regular measure $m: \mathscr{B}(S) \rightarrow F^{\prime}$ is bounded if $\|m(A)\|$ is a bounded function of $A$. It follows from [7, p. 97, Lemma 5] that, if $m$ is bounded, then

$$
\|m\|=\sup \overline{\mu_{y}}(S)<+\infty
$$

where the sup in (3.1) is taken for $\|y\| \leqq 1$. The space $N\left(S, F^{\prime}\right)$ of all bounded weak* regular measures $m: \mathscr{B}(S) \rightarrow F^{\prime}$ is a Banach space under the norm 
(3.1), which we call the semivariation of $m$. The same is true about the space $N_{s}(S, F)$ of weak regular measures $m: \mathscr{B}(S) \rightarrow F$. For these measures, our notion of semivariation reduces to that of [2]. A measure $m \in N_{s}(S, F)$ is not only weakly countably additive, but also strongly countably additive, hence the subscript $s$ in $N_{s}(S, F)$. The following theorem is an easy extension of $[2$, p. 300 , Theorem 3.1]. Its proof is omitted.

THEOREM 3.1. There exists an isometric isomorphism, symbolized by the equation

$$
\mathscr{L}\left[C(S), F^{\prime}\right]=N\left(S, F^{\prime}\right)
$$

in such a way that, if $m^{V} \in N\left(S, F^{\prime}\right)$ corresponds to $V \in \mathscr{L}\left[C(S), F^{\prime}\right]$ under this isometry, then for every $\phi \in C(S)$,

$$
V \phi=\int_{S} \phi(s) d m^{V}
$$

Let now $m \in N_{s}(S, F)$. For the definition of the (scalar) variation measure of $m$ we refer the reader to [5]. We denote it by $\mu^{m}$. We denote by $M(S, F)$ the vector space of all $m \in N_{s}(S, F)$ of bounded variation, i.e., $\mu^{m}(S)<+\infty$. The expression $\mu^{m}(S)$ defines a norm on $M(S, F)$ under which it is a Banach space.

We now borrow a term from Grothendieck [10, p. 104, Definition 6], to describe a concept, closely related to his, but apparently not equivalent. We say that a Banach space $E$ is a Phillips space if for every compact Hausdorff space $S$, every Radon measure $\mu$ on $S$ and every bounded linear transformation $T: L_{1}(\mu) \rightarrow F$, there exists a strongly measurable mapping $f: S \rightarrow F$ such that ess $\sup _{s \in S}\|f(s)\|=\|T\|$ and, for every $\phi \in L_{1}(\mu)$,

$$
T_{\phi}=\int_{S} \phi(s) f(s) d \mu .
$$

It follows from the discussion in [10] (even when we use the present definition) that $F$ is a Phillips space if it is reflexive and that $F^{\prime}$ is a Phillips space if it is separable. Although a vector measure of bounded variation is absolutely continuous with respect to its variation measure, there does not exist, in general, a "Radon-Nikodym" theorem relating them. However the. following theorem is valid for Phillips spaces. It seems to be in the same spirit, but logically independent of several other theorems already in the literature. For some of these, see $[12$, p. 81 , Corollary 2$],[13$, pp. 788789, Theorems 5 and 6], and [3, p. 47, Corollary 4].

Theorem 3.2. Let $E$ be a Phillips space and $m: \mathscr{B}(S) \rightarrow E$ a regular measure of bounded variation. Then there exists a strongly measurable mapping $g^{m}$ : $S \rightarrow E$, with $\left\|g^{m}\right\|=1$, identically, so that for every $f \in L_{1}\left(\mu^{m}, E^{\prime}\right)$, we have 


$$
\int_{S}\langle d m, f(s)\rangle=\int_{S}\left\langle g^{m}(s), f(s)\right\rangle d^{m} .
$$

Proof. For the definition and basic properties of the integral on the left of (3.5) see [5]. Define $T_{\phi}=\int_{S} \phi(s) d m$, for $\phi \in L_{1}\left(\mu^{m}\right), T: L_{1}\left(\mu^{m}\right) \rightarrow E$, and $\|T\| \leqq 1$. Since $E$ is a. Phillips space, there exists a strongly measurable $g^{m}: S \rightarrow E$ with $\operatorname{ess} \sup _{s \in S}\left\|g^{m}(s)\right\| \leqq 1$ such that, for every $\phi \in L_{1}\left(\mu^{m}\right)$,

$$
T_{\phi}=\int_{S} \phi(s) g^{m}(s) d \mu^{m}
$$

Direct substitution shows that (3.5) follows from (3.6) for simple functions $f(s)=\sum_{i=1}^{n} \chi_{A_{i}}(s) x_{i}^{\prime}, A_{i} \in \mathscr{B}(S)$ and $x_{i}^{\prime} \in E^{\prime}$. Then (3.5) can be extended to every $f \in L_{1}\left(\mu^{m}, E^{\prime}\right)$. It remains to show that $\left\|g^{m}(s)\right\|=1$ almost everywhere. If not, for some $\epsilon<1$ and $\epsilon>0$ there exists $A \in \mathscr{B}(S)$, with $\mu^{m}(A)>0$, so that $\left\|g^{m}(s)\right\|<\epsilon$ for $s \in A$. Then, for any disjoint decomposition $A=\bigcup_{i=1}^{n} A_{i}, A_{i} \in \mathscr{B}(S)$, we have

$$
\begin{aligned}
\sum_{i=1}^{n}\left\|m\left(A_{i}\right)\right\| & =\sum_{i=1}^{n}\left\|\int_{S} \chi_{A_{i}}(s) d m\right\|=\sum_{i=1}^{n}\left\|\int_{A_{i}} g^{m}(s) d \mu^{m}\right\| \\
& \leqq \sum_{i=1}^{n} \int_{A_{i}}\left\|g^{m}(s)\right\| d \mu^{m} \leqq \epsilon \mu^{m}(A) .
\end{aligned}
$$

From (3.7) and the definition of $\mu^{m}$, it follows that $0<\mu^{m}(A) \leqq \epsilon \mu^{m}(A)$, a contradiction. This completes the proof.

4. Tensor products of measure spaces. The formula (2.1) for $t \in C^{\prime}(S)$ $\otimes F$ becomes

$$
t=\sum_{j=1}^{n} \mu_{j} \otimes y_{j}
$$

where $\mu_{j} \in C^{\prime}(S)$ and $y_{j} \in F$. Formula (2.2) for $\phi \in C(S)$ becomes

$$
U^{t} \phi=\sum_{j=1}^{n} y_{j} \int_{S} \phi(s) d \mu_{j}
$$

It has been shown by Grothendieck [10, p. 185, Proposition 41] that $C^{\prime}(S)$ satisfies the condition of metric approximation, a matter which will not be discussed here in detail. For another discussion of this property see [9]. We draw two consequences from this property (see [10, p. 164, Proposition 36]). One is that $C^{\prime}(S) \otimes_{\lambda} F$ can be identified with the space of all compact linear transformations from $C(S)$ into $F$. The other is that the mapping $\Phi_{\gamma}^{\lambda} . C^{\prime}(S) \otimes_{\gamma} F \rightarrow C^{\prime}(S) \otimes_{\lambda} F$ is one-to-one, i.e., the correspondence $t \rightarrow U^{t}$, 
$t \in C^{\prime}(S) \otimes_{\gamma} F$ is one-to-one. This means that $C^{\prime}(S) \otimes_{\gamma} F=C^{\prime}(S) \otimes_{\tau} F$ isometrically.

We are going to study both spaces $C^{\prime}(S) \otimes_{\gamma} F$ and $C^{\prime}(S) \otimes_{\lambda} F$, although our primary interest is in $C^{\prime}(S) \otimes_{\gamma} F$. We do this because there are many twin results for these spaces. In each pair the result concerning $C^{\prime}(S) \otimes_{\gamma} F$ is usually the harder of the two, while the result about $C^{\prime}(S) \otimes_{\lambda} F$ is either easy or in the literature in one form or another. Yet it is educational to exhibit the parallel results together when they occur. It turns out that each of these tensor products can be identified with a space of vector measures.

Theorem 4.1. There exists an isometry of $C^{\prime}(S) \otimes_{\lambda} F$ into $N_{s}(S, F)$ in such a way that if, under this isometry $m^{t} \in N_{s}(S, F)$ corresponds to $t \in C^{\prime}(S) \otimes F$, with representation (4.1), then for $A \in \mathscr{B}(S)$, we have

$$
m^{t}(A)=\sum_{j=1}^{n} \mu_{j}(A) y_{j}
$$

If $m^{t}$ corresponds to $t \in C^{\prime}(S) \otimes_{\lambda} F$ with representation (2.4), then

$$
m^{t}=\sum_{k=1}^{\infty} m^{t_{k}}
$$

where the series converges absolutely in $N_{s}(S, F)$. For every $\phi \in C(S)$

$$
U^{t} \phi=\int_{S} \phi(s) d m^{t}
$$

Proof. For $t \in C^{\prime}(S) \otimes F$, represented by (4.1), $m^{t}$ is well defined by (4.3) and substitution in (4.5) yields equality readily. We have $\lambda(t)=\left\|U^{t}\right\|$ and, by Theorem 3.1 this norm is $\left\|m^{t}\right\|$. For general $t \in C^{\prime}(S) \otimes_{\lambda} F$ represented by (2.4) one shows that $m^{t}$ given by (4.4) satisfies (4.5) by the same argument one uses in establishing the convergence of the series in (4.4).

THEOREM 4.2. There exists an isometric isomorphism of $C^{\prime}(S) \otimes_{\gamma} F$ into $M(S, F)$ in such a way that, if under this isomorphism, $m^{t} \in M(S, F)$ corresponds to $t \in C^{\prime}(S) \otimes_{\gamma} F$, and $t$ is represented by (2.4), then $m^{t}$ is represented by (4.4) and $U^{t}$ by (4.5).

Proof. This theorem is a consequence of Corollary 2, p. 371, of [11]. The following is a direct proof. Let $t \in C^{\prime}(S) \otimes_{\gamma} F$. Since $t \in C^{\prime}(S) \otimes_{\lambda} F$ also, (4.4) defines $m^{t} \in N_{s}(S, F)$ satisfying (4.5). It follows from (4.4) that $\mu^{m t}(S) \leqq \gamma(t)$. We show the reverse inequality. It suffices to do this for finite tensors $t=\sum_{j=1}^{n} \mu_{j} \otimes y_{j}$. Let $\nu$ be a non-negative regular measure on $S$ with respect to which every $\mu_{j}$ is absolutely continuous. There is an isometry $T$ of $L_{1}(\nu)$ into $C^{\prime}(S)$ and functions $\phi_{1}, \phi_{2}, \cdots, \phi_{n} \in L_{1}(\nu)$ such that $\mu_{j}=T_{\phi_{j}}$ for every $j$. For every $A \in \mathscr{B}(S)$, 


$$
m^{t}(A)=\sum_{j=1}^{n}\left[\int_{A} \phi_{j}(s) d \nu\right] y_{j}=\int_{A} f(s) d \nu,
$$

where $f(s)=\sum_{j=1}^{n} \phi_{j}(s) y_{j}$. Let $\bar{\gamma}$ be the greatest cross norm of $L_{1}(\nu) \otimes F$. Since $T$ is an isometry, for any $t \in L_{1}(\nu) \otimes_{\bar{\gamma}} F, \bar{\gamma}(t) \geqq \gamma\left(t_{1}\right)$, where $t_{1}$ is the tensor in $C^{\prime}(S) \otimes_{\gamma} F$ corresponding to $t$. Then, from (4.5) we get by using (2.16),

$$
\mu^{m^{t}}(S)=\int_{S}\|f(s)\| d \nu=\bar{\gamma}\left(\sum_{j=1}^{n} \phi_{j} \otimes y_{j}\right) \geqq \gamma(t) .
$$

This completes the proof.

Theorem 4.3. A measure $m \in N_{s}(S, F)$ is of the form $m^{t}$ for $t \in C^{\prime}(S) \otimes_{\lambda} F$ if and only if $V^{m}: C(S) \rightarrow F$, defined by

$$
V^{m} \phi=\int_{S} \phi(s) d m
$$

for $\phi \in C(S)$, is a compact transformation.

Proof. Taking account of the isometric inclusions $C^{\prime}(S) \otimes_{\lambda} F \subset N_{s}(S, F)$ $\subset \mathscr{L}[C(S), F]$, the statement of the theorem can be translated into the statement that $U: C(S) \rightarrow F$ is a compact linear transformation if and only if $U=U^{t}$ for some $t \in C^{\prime}(S) \otimes_{\lambda} F$.

Theorem 4.4. If $F$ is a Phillips space, then

$$
C^{\prime}(S) \otimes_{\gamma} F=M(S, F)
$$

isometrically.

Proof. In view of Theorem 4.2, it suffices to show that every $m \in M(S, F)$ is of the form $m^{t}$, for $t \in C^{\prime}(S) \otimes_{\gamma} F$. So let $m \in M(S, F)$. By Theorem 3.2, there exists a strongly measurable mapping $g: S \rightarrow F$ ' so that $\|g(s)\|=1$, identically and so that, for every $f \in L_{1}\left(\mu^{m}, F^{\prime}\right)$, we have

$$
\int_{S}\langle d m, f(s)\rangle=\int_{S}\langle g(s), f(s)\rangle d \mu^{m}
$$

It follows from (4.9) that for every $A \in \mathscr{B}(S), m(A)=\int_{A} g(s) d_{\mu}{ }^{m}$. Now $g \in L_{1}\left(\mu^{m}, F\right)=L_{1}\left(\mu^{m}\right) \otimes_{\gamma} F$. Hence $g$ is defined by a tensor $t_{0} \in L_{1}\left(\mu^{m}\right) \otimes_{\gamma} F$ with a representation $(2.10)$ of the form $\sum_{j=1}^{\infty} \phi_{j} \otimes y_{j}, \phi_{j} \in L_{1}\left(\mu^{m}\right), y_{j} \in F$, with $\sum_{j=1}^{\infty}\left\|\phi_{j}\right\|_{1}\left\|y_{j}\right\|<+\infty$. For each $j$ let $\mu_{j}$ be the regular measure on $S$ whose Radon-Nikodym derivative with respect to $\mu^{m}$ is $\phi_{j}$. Then it is easy to see that $m=m^{t}$ for $t \in C^{\prime}(S) \otimes_{\gamma} F$ with a representation (2.10) given by $\sum_{j=1}^{\infty} \mu_{j} \otimes y_{j}$. 
5. Duality of tensor products. In this section we gather a few consequences of the results of $\$ 4$ having to do with the duality of $E \otimes_{\lambda} F$ and $E \otimes_{\gamma} F$. Some of the results given in this section are due to Grothendieck, but their proofs as consequences of the above discussion of vector valued measures appear more natural and elementary. We begin with $C(S) \otimes_{\lambda} F$ and $C(S) \otimes_{\gamma} F$. By (2.15) we know that to any $t \in C(S) \otimes_{\lambda} F$ corresponds a unique continuous mapping $f^{t}: S \rightarrow F$ in the following manner. For $t \in C(S) \otimes F$ represented by

$$
t=\sum_{j=1}^{n} \phi_{j} \otimes y_{j}
$$

$\phi_{j} \in C(S), y_{j} \in F$, we have for $s \in S$

$$
f^{t}(s)=\sum_{j=1}^{n} \phi_{j}(s) y_{j}
$$

and for $t \in C(S) \otimes_{\lambda} F$ represented by (2.4),

$$
f^{t}=\sum_{k=1}^{\infty} f^{t_{k}}
$$

where the series converges absolutely in $C(S, F)$ and $\lambda(t)=\left\|f^{t}\right\|_{\infty}$. Now for $t \in C(S) \otimes_{\gamma} F$ we still have a mapping $f^{t}$, namely the one given by (5.3) for $\Phi_{\gamma}^{\lambda}(t) \in C(S) \otimes_{\lambda} F$. But in this case an explicit expression for $t^{t}$ is easier to write. It is given, for $s \in S$, by

$$
f^{t}(s)=\sum_{j=1}^{\infty} \phi_{j}(s) y_{j}
$$

if

$$
t=\sum_{j=1}^{\infty} \phi_{j} \otimes y_{j}
$$

is the representation (2.10) of $t$.

The symbol $\delta_{S}\langle f(s), d m\rangle$ has been used up to this point in our discussion, to denote an integral for $f \in L_{1}\left(\mu^{m}, F\right)$ and $m \in M\left(S, F^{\prime}\right)$ or for inessential variations of this setting. Now we need the same integral for $f$ replaced by $f^{t}$, given by (5.4) and for $m \in N\left(S, F^{\prime}\right)$. This integral $\left({ }^{2}\right)$ can be given a meaning as part of a general theory of integration [1]. However, for the sake of simplicity, let us accept the following equation as a definition:

$\left(^{2}\right)$ This integral $\int_{S}\left\langle f^{t}(s), d m\right\rangle$ is the analogue of the Stieltjes integral $\int_{\dot{a}}^{b} f(s) d g(s)$ for $g$ continuous and $f$ of bounded variation. 


$$
\int_{S}\left\langle f^{t}(s), d m\right\rangle=\sum_{j=1}^{\infty}\left\langle y_{j}, \int_{S} \phi_{j}(s) d m\right\rangle .
$$

Theorem 5.1. There is an isometric isomorphism, represented by the following equation

$$
\left[C(S) \otimes_{\gamma} F\right]^{\prime}=N\left(S, F^{\prime}\right),
$$

such that if $m \in N\left(S, F^{\prime}\right)$ corresponds to $t^{\prime} \in\left[C(S) \otimes_{\gamma} F\right]^{\prime}$ under this isometry, then for every $t \in C(S) \otimes_{\gamma} F$,

$$
\left\langle t, t^{\prime}\right\rangle=\int_{S}\left\langle f^{t}(s), d m\right\rangle .
$$

Proof. If we put (2.12) and Theorem 3.1 together, we obtain the relations $\left[C(S) \otimes_{\gamma} F\right]^{\prime}=\mathscr{L}\left[C(S), F^{\prime}\right]=N\left(S, F^{\prime}\right)$. If under these identifications $T \in \mathscr{L}\left[C(S), F^{\prime}\right]$ and $m \in N\left(S, F^{\prime}\right)$ correspond to $t^{\prime}$, we have for every $t \in C(S) \otimes_{\gamma} F$, represented by $(5.5)$,

$$
\left\langle t, t^{\prime}\right\rangle=\sum_{j=1}^{\infty}\left\langle y_{j}, T \phi_{j}\right\rangle=\int_{S}\left\langle f^{t}(s), d m\right\rangle .
$$

The last equality of (5.9) is obtained from (3.3) in Theorem 3.1 and (5.6).

TheOREM 5.2. There exists an isometric isomorphism represented by the equation

$$
\left[C(S) \otimes_{\lambda} F\right]^{\prime}=M\left(S, F^{\prime}\right)
$$

such that if $m \in M\left(S, F^{\prime}\right)$ corresponds to $t^{\prime} \in\left[C(S) \otimes_{\lambda} F\right]^{\prime}$ under this isometry, then, for any $t \in C(S) \otimes_{\lambda} F,\left\langle t, t^{\prime}\right\rangle$ is represented by (5.8).

Proof. The proof of this theorem is similar to that of the previous theorem and follows from the corresponding relations $C(S) \otimes_{\lambda} F=C(S, F)$ (see (2.15)), and a theorem of Ivan Singer [17] stating that $C^{\prime}(S, F)=M\left(S, F^{\prime}\right)$, where $C^{\prime}(S, F)$ is the dual space of $C(S, F)$.

Corollary 5.1 (Grothendieck). If $F^{\prime}$ is a Phillips space, then $\left[C(S) \otimes_{\lambda} F\right]^{\prime}$ $=C^{\prime}(S) \otimes_{\gamma} F^{\prime}$, isometrically.

Proof. This is a direct consequence of Theorem 16 and Theorem 14 . It should be remembered that if $F^{\prime}$ is separable or reflexive, then it is a Phillips space.

We now turn to the duality of $E \otimes_{\lambda} F$ in a more general setting. The proof of the following theorem, which seems to be new, leans heavily on methods scattered throughout [10].

Theorem 5.3. If either $E^{\prime}$ or $F^{\prime},\left(\right.$ say $\left.F^{\prime}\right)$, is a Phillips space, then $\left(E \otimes_{\lambda} F\right)^{\prime}$ $=E^{\prime} \otimes_{\tau} F^{\prime}$, isometrically. 
Proof. See $§ 2$ for the definition and properties of $\tau$. We know that $E^{\prime} \otimes_{\tau} F^{\prime}$ can be identified with the subset of $\mathscr{L}\left(E, F^{\prime}\right)$ consisting of nuclear transformations. Let $t^{\prime} \in E^{\prime} \otimes_{\gamma} F^{\prime}$ and $U^{\prime}: E \rightarrow F^{\prime}$ be the nuclear transformation that corresponds to $t^{\prime}$. If $t^{\prime}$ is represented by

$$
t^{\prime}=\sum_{j=1}^{\infty} x_{j}^{\prime} \otimes y_{j}^{\prime}
$$

with $x_{j}^{\prime} \in E^{\prime}, y_{j}^{\prime} \in F^{\prime}$ and $\sum_{j=1}^{\infty}\left\|x_{j}^{\prime}\right\|\left\|y_{j}^{\prime}\right\|<+\infty, U^{\prime}$ is given, for $x \in E$, by $U^{\prime} x=\sum_{j=1}^{\infty}\left\langle x, x_{i}^{\prime}\right\rangle y_{i}^{\prime}$, where the series converges absolutely in $F^{\prime}$. We also know that $\tau\left(t^{\prime}\right)=\inf \sum_{j=1}^{\infty}\left\|x_{j}^{\prime}\right\|\left\|y_{j}^{\prime}\right\|$, where the inf is taken over all representations (5.10) of $t^{\prime}$. Let $t \in E \otimes F$ be represented by

$$
t=\sum_{i=1}^{n} x_{i} \otimes y_{i}
$$

$x_{i} \in E$ and $y_{i} \in F$. We set

$$
\begin{aligned}
\left\langle t, t^{\prime}\right\rangle & =\sum_{i=1}^{n} \sum_{j=1}^{\infty}\left\langle x_{i}, x_{j}^{\prime}\right\rangle\left\langle y_{i}, y_{j}^{\prime}\right\rangle \\
& =\sum_{i=1}^{n}\left\langle y_{i}, U^{t} x_{i}\right\rangle .
\end{aligned}
$$

It is easy to see that the value of $\left\langle t, t^{\prime}\right\rangle$ is independent of the choice of representation (5.10) of $t^{\prime}$, hence is uniquely determined by $U^{t}$. What is more, one can easily show that for any representation (5.10) we have $\left|\left\langle t, t^{\prime}\right\rangle\right|$ $\leqq \lambda(t) \sum_{j=1}^{\infty}\left\|x_{j}^{\prime}\right\|\left\|y_{j}^{\prime}\right\|$. Hence $\left|\left\langle t, t^{\prime}\right\rangle\right| \leqq \lambda(t)_{\tau}\left(t^{\prime}\right)$. It follows that every $t^{\prime}$ $\in E^{\prime} \otimes_{r} F^{\prime}$ can be identities with a bounded linear functional on $E \otimes F$ which can be extended to a unique bounded linear functional on $E \otimes_{\lambda} F$ and that when we consider $t^{\prime}$ as such a functional we have $\lambda^{\prime}\left(t^{\prime}\right) \leqq \tau\left(t^{\prime}\right)$. We will have completed our proof when we have shown that every bounded linear functional on $E \otimes_{\lambda} F$ is given by (5.12) and that $\lambda^{\prime}\left(t^{\prime}\right) \geqq \tau\left(t^{\prime}\right)$. We proceed to do this.

Let $S$ be the solid unit sphere of $E^{\prime}$ under the weak* topology. Since each $x \in E$ can be considered as a scalar valued function on $S$, it is possible to imbed $E \otimes_{\lambda} F$ isometrically in $C(S, F)=C(S) \otimes_{\lambda} F$. The formulas for this imbedding can be written down as follows. Let

$$
t=\sum_{i=1}^{n} x_{i} \otimes y_{i}
$$

where $x_{i} \in E, y_{i} \in F$. To $t$ we assign the mapping $f^{t}: S \rightarrow F$ given, for $x^{\prime} \in S$, by 


$$
f^{t}\left(x^{\prime}\right)=\sum_{i=1}^{n}\left\langle x_{i}, x^{\prime}\right\rangle y_{i}
$$

If $t \in E \otimes_{\lambda} F$ is represented by (2.4) then we define $f^{t}$ by means of (5.3) and (5.14). It is easy to see that the assignment $t \leftrightarrow f^{t}$ is an isometry of $E \otimes_{\lambda} F$ into $C(S, F)$. Let now $t^{\prime} \in\left(E \otimes_{\lambda} F\right)^{\prime}$. By the Hahn-Banach theorem, there exists a unique $q^{\prime} \in C^{\prime}(S, F)$ so that $\left\|q^{\prime}\right\|=\lambda^{\prime}\left(t^{\prime}\right)$ and $\left\langle t, t^{\prime}\right\rangle=\left\langle f^{t}, q^{\prime}\right\rangle$ for every $t \in E \otimes_{\lambda} F$. Under our hypothesis (Corollary 5.1) we have $C^{\prime}(S, F)$ $=C^{\prime}(S) \otimes_{\gamma} F^{\prime}$. It follows that $q^{\prime}$ can be identified with a tensor with representation

$$
q^{\prime}=\sum_{j=1}^{\infty} \mu_{j} \otimes y_{j}^{\prime},
$$

where $\sum_{j=1}^{\infty} \bar{\mu}_{j}(S)\left\|y_{j}^{\prime}\right\|<+\infty$. Then, for $t \in E \otimes F$, represented by (5.13) we have

$$
\left\langle t, t^{\prime}\right\rangle=\sum_{j=1}^{\infty} \sum_{i=1}^{n}\left\langle y_{i}, y_{j}^{\prime}\right\rangle \int_{S}\left\langle x_{j}, x^{\prime}\right\rangle d \mu_{j}\left(x^{\prime}\right) .
$$

Let now

$$
x_{j}^{\prime}=\int_{S} x^{\prime} d \mu_{j}\left(x^{\prime}\right)
$$

Then

$$
\left\langle t, t^{\prime}\right\rangle=\sum_{j=1}^{\infty} \sum_{i=1}^{n}\left\langle x_{i}, x_{j}^{\prime}\right\rangle\left\langle y_{i}, y_{j}^{\prime}\right\rangle .
$$

Since $\sum_{j=1}^{\infty}\left\|x_{j}^{\prime}\right\|\left\|y_{j}^{\prime}\right\| \leqq \sum_{j=1}^{\infty} \bar{\mu}_{j}(S)\left\|y_{j}^{\prime}\right\|<+\infty$, we see that $t^{\prime}$ arises from the tensor $\sum_{j=1}^{\infty} x_{j}^{\prime} \otimes y_{j}^{\prime} \in E^{\prime} \otimes_{\tau} F^{\prime}$. Consequently the imbedding of $E^{\prime} \otimes_{\tau} F^{\prime}$ into $\left(E \otimes_{\lambda} F^{\prime}\right)^{\prime}$ described in the first part of this proof is onto. It remains to show that $\lambda^{\prime}\left(t^{\prime}\right) \geqq \tau\left(t^{\prime}\right)$. Since any representation (5.15) of $q^{\prime}$ yields a corresponding representation of $t^{\prime}$, we have $\gamma\left(q^{\prime}\right) \geqq \tau\left(t^{\prime}\right)$. Then $\lambda^{\prime}\left(t^{\prime}\right)=\gamma\left(q^{\prime}\right)$ $\geqq \tau\left(t^{\prime}\right)$. Q.E.D.

Corollary 5.2 (Grothendieck). If either $E^{\prime}$ or $F^{\prime}$ satisfies the condition of approximation of Grothendieck (see \$4) and if either $E^{\prime}$ or $F^{\prime}$ is a Phillips space, then $\left(E \otimes_{\lambda} F\right)^{\prime}=E^{\prime} \otimes_{\gamma} F^{\prime}$, isometrically.

Proof. From the fact that one of the spaces $E^{\prime}$ and $F^{\prime}$ is a Phillips space, it follows by Theorem 5.3 that $\left(E \otimes_{\lambda} F\right)^{\prime}=E^{\prime} \otimes_{\tau} F^{\prime}$, isometrically. Our present corollary follows from the fact that if one of the spaces $E^{\prime}$ and $F^{\prime}$ satisfies the condition of approximation, then $E^{\prime} \otimes_{\gamma} F^{\prime}=E^{\prime} \otimes_{\tau} F^{\prime}$, isometrically (see $\$ 4$ for details). 


\section{Part II. Tensor Products of Spaces with Schauder Bases}

6. Unconditional Schauder bases. Let $\Omega=\left\{y_{i}, y_{i}^{\prime}\right\}, y_{i} \in F, y_{i}^{\prime} \in F^{\prime}$, be a biorthogonal system. We say that $\Omega$ defines an unconditional (Schauder) basis for $F$ if for every $y \in F$,

$$
y=\sum_{i=1}^{\infty}\left\langle y, y_{i}^{\prime}\right\rangle y_{i}
$$

and the series on the right of (6.1) converges unconditionally. For a general discussion of bases and of unconditional convergence consult [4, Chapter IV]. For a discussion of bases of tensor products see [8]. Let $\Omega^{\prime}=\left\{y_{i}^{\prime}, y_{i}\right\}$, and consider each $y_{i}$ as an element of $F^{\prime \prime}$. $\Omega^{\prime}$ does not in general define a basis for $F^{\prime}$ in the ordinary sense, but it does define what we shall call a weak* basis for $F^{\prime}$, which will quite suffice for our purposes. By this we mean that for every $y^{\prime} \in F^{\prime}$,

$$
y^{\prime}=* \sum_{i=1}^{\infty}\left\langle y_{i}, y^{\prime}\right\rangle y_{i}^{\prime}
$$

where the infinite series converges in the weak* topology. This type of convergence is signified by the star next to the = sign. We say that the norm of $F$ is basic with respect to $\Omega$ if it is given, for every $y \in F$, by

$$
\|y\|=\sup \sum_{i=1}^{\infty}\left|\left\langle y, y_{i}^{\prime}\right\rangle\right|\left|\left\langle y_{i}, y^{\prime}\right\rangle\right|
$$

where the sup is taken for all $y^{\prime} \in F^{\prime},\left\|y^{\prime}\right\| \leqq 1$. Similarly we say that the norm of $F^{\prime}$ is basic with respect to $\Omega^{\prime}$ if, for $y^{\prime} \in F^{\prime},\left\|y^{\prime}\right\|$ is given by the sup on the right of (6.3) for all $y \in F,\|y\| \leqq 1$. It is not hard to see that the norm of $F$ is basic with respect to $\Omega$ if and only if its dual norm is basic with respect to $\Omega^{\prime}$. It is also easy to verify that the standard Schauder bases for $c_{0}, l_{p}$, $1 \leqq p<+\infty$, and any orthonormal basis of separable Hilbert space are unconditional bases and that the norms of each of these spaces is basic with respect to the corresponding biorthogonal system. Actually the main result of this section states that for any Banach space $F$, for which $\Omega$ defines an unconditional Schauder basis, its norm can be replaced by an equivalent norm which is basic with respect to $\Omega$. To prove this result we utilize some of the properties of $l_{1} \otimes_{\lambda} F$. Perhaps a proof can be given without the use of tensor products, but the present proof is more in keeping with the spirit of this work and some of the required properties of $l_{1} \otimes_{\lambda} F$ have some intrinsic interest.

It has been shown by Grothendieck [10, pp. 90-92] that the space $l_{1} \otimes_{\lambda} F$ 
can be identified with the space $\mathscr{U}(F)$ of all unconditionally summable $F$-sequences, i.e., all sequences $u_{1}, u_{2}, \cdots, u_{i} \in F$, so that all permutations of the series $\sum_{i=1}^{\infty} u_{i}$ converge to some element of $F$. The following lemma gives an explicit expression for $\lambda$ in this case.

Lemma 6.1. Let $t=\left\{u_{1}, u_{2}, \cdots\right\} \in \mathscr{U}(F)=l_{1} \otimes_{\lambda} F$. Then

$$
\lambda(t)=\sup \sum_{i=1}^{\infty}\left|\left\langle u_{i}, y^{\prime}\right\rangle\right|,
$$

where the sup is taken for all $y^{\prime} \in F^{\prime}$, with $\left\|y^{\prime}\right\| \leqq 1$.

Proof. By definition, the inclusion $l_{1} \otimes_{\lambda} F \subset \mathscr{L}\left(l_{\infty}, F\right)$ is an isometry. It follows from the definition of $U^{t}$ (see (2.5)) that, for $\mathscr{S}=\left\{\sigma_{1}, \sigma_{2}, \ldots\right\} \in l_{\infty}$ $=\left(l_{1}\right)^{\prime}, U^{t} \mathscr{Y}=\sum_{i=1}^{\infty} \sigma_{i} u_{i}$. Then, taking all sups for all $y^{\prime} \in F^{\prime},\left\|y^{\prime}\right\| \leqq 1$, and all $\mathscr{S}$, with every $\left|\sigma_{i}\right| \leqq 1$, we have

$$
\lambda(t)=\sup \left\|U^{t} \mathscr{S}\right\|=\sup \left\langle U^{t} \mathscr{S}, y^{\prime}\right\rangle=\sup \sum_{i=1}^{\infty} \sigma_{i}\left\langle u_{i}, y^{\prime}\right\rangle
$$

$$
\leqq \sup \sum_{i=1}^{\infty}\left|\left\langle u_{i}, y^{\prime}\right\rangle\right|
$$

To show the converse inequality we note that each $\left|\left\langle u_{i}, y^{\prime}\right\rangle\right|=\sigma_{i}\left\langle u_{i}, y^{\prime}\right\rangle$, for suitable $\sigma_{i}$ with $\left|\sigma_{i}\right|=1$. If $\mathscr{S}=\left\{\sigma_{1}, \sigma_{2}, \cdots\right\}$ then $\sum_{i=1}^{\infty}\left|\left\langle u_{i}, y^{\prime}\right\rangle\right|$ $=\left\langle U^{t} \mathscr{Y}, y^{\prime}\right\rangle \leqq \lambda(t)$. From this and (6.5) we obtain (6.4).

Theorem 6.1. If $\Omega$ defines an unconditional Schauder basis for $F$, then there exists a norm of $F$, basic with respect to $\Omega$, which is equivalent to the original norm of $F$.

Proof. We define a linear transformation, $T: F \rightarrow \mathscr{U}(F)$ by $T y$ $=\left\{\left\langle y, y_{1}^{\prime}\right\rangle y_{1},\left\langle y, y_{2}^{\prime}\right\rangle y_{2}, \cdots\right\}$, for $y \in F$ represented by (6.1). Clearly $T$ is an algebraic open isomorphism. We show that $T(F)$ is a closed subspace of $\mathscr{U}(F)$. Suppose that $t^{j}=T y^{j}$, and that the sequence $t^{1}, t^{2}, \ldots$ converges to some $t=\left\{u_{1}, u_{2}, \cdots\right\} \in \mathscr{U}(F)$. It can be easily seen that, for every $i$,

$$
\left\|u_{i}-\left\langle y^{j}, y_{i}^{\prime}\right\rangle y_{i}\right\| \leqq \lambda\left(t-t^{j}\right) \rightarrow 0
$$

as $j \rightarrow \infty$. Consequently for every $i, u_{i}=\theta_{i} y_{i}$ for some scalar $\theta_{i}$, and $t=T y$ for $y=\sum_{i=1}^{\infty} \theta_{i} y_{i}$. Hence $T(F)$ is closed. By the open mapping theorem $T$ is a homeomorphism. We now define, for every $y \in F,\|y\|_{\Omega}=\lambda(T y)$. It is easy to verify that \|\|$_{\Omega}$ satisfies (6.3) and is basic with respect to $\Omega$. It is equivalent to \|\| . This completes our proof.

Compare \|\|$_{\Omega}$ with a similar norm obtained in $[4$, p. 73 , Theorem 1$]$. 
7. Tensor products of spaces with unconditional Schauder bases. We are now ready to investigate further the tensor product $E \otimes_{\gamma} F$ in the case in which $F$ has an unconditional Schauder basis. Suppose that $\Omega=\left\{y_{i}, y_{i}^{\prime}\right\}$ is as in $\$ 6$ and defines such a basis for $F$. In view of Theorem 6.1 one can, and will, without loss of generality, assume that the norm of $F$ is basic with respect to $\Omega$. It follows that the dual norm is basic with respect to $\Omega^{\prime}$.

It is well known [14] that any compact linear transformation with range in $F$ can be approximated uniformly by linear transformations of finite rank. This means in the terminology of Grothendieck that $F$ satisfies the condition of approximation. From this we can draw two consequences that are important in what follows (see numbers 3 and 4 of the corollary on p. 168 of [10]). One is that $E \otimes_{\lambda} F$ can be identified with the space of all compact linear transformations of $E^{\prime}$ into $F$ that are continuous with respect to the weak* topology of $E^{\prime}$ and the weak topology of $F$. The other consequence is that the mapping $\Phi_{\gamma}^{\lambda}: E \otimes_{\gamma} F \rightarrow E \otimes_{\lambda} F$ is one-to-one. Consequently $E \otimes_{\tau} F$ $=E \otimes_{\gamma} F \subset E \otimes_{\lambda} F \subset \mathscr{L}\left(E^{\prime}, F\right)$. Now we know that, for every $t \in E \otimes_{\gamma} F$, $U^{t}$ is a compact transformation. However it seems to be a very difficult problem to determine just which compact transformations are nuclear, i.e., of the form $U^{t}, t \in E \otimes_{\gamma} F$. The next theorem gives a partial answer under the assumptions of this section. We begin with some preliminary material.

Let $U: E^{\prime} \rightarrow F$ be a bounded linear transformation which is continuous with respect to the weak ${ }^{*}$ topology of $E^{\prime}$ and the weak topology of $F$. For each $i$, the expression $\left\langle U x^{\prime}, y_{i}^{\prime}\right\rangle, x^{\prime} \in E^{\prime}$ defines a weakly* continuous linear functional on $E^{\prime}$, hence an element $x_{i}$ of $E$, such that

$$
\left\langle x_{i}, x^{\prime}\right\rangle=\left\langle U x^{\prime}, y_{i}^{\prime}\right\rangle \text {. }
$$

This yields the following representation for $U$,

$$
U x^{\prime}=\sum_{i=1}^{\infty}\left\langle x_{i}, x^{\prime}\right\rangle y_{i}
$$

In particular, for $t \in E \otimes_{\gamma} F, U^{t}$ has a representation (7.2).

Let now $t \in E \otimes_{\gamma} F$. Then, as in $\S 2$, we have

$$
t=\sum_{j=1}^{\infty} u_{j} \otimes v_{j}
$$

with $u_{j} \in E$ and $v_{j} \in F$ for every $j$, with

$$
\sum_{j=1}^{\infty}\left\|u_{j}\right\|\left\|v_{j}\right\|<+\infty .
$$

Then, for every $t \in E \otimes_{\gamma} F, U^{t}$ (see (2.5) above) is given by 


$$
U^{t} x^{\prime}=\sum_{j=1}^{\infty}\left\langle u_{j}, x^{\prime}\right\rangle v_{j}
$$

for every $x^{\prime} \in E^{\prime}$.

Lemma 7.1. Let $t \in E \otimes_{\gamma} F$ be represented by (7.3). Then, for every $i$, the series in

$$
x_{i}=\sum_{j=1}^{\infty}\left\langle v_{j}, y_{i}^{\prime}\right\rangle u_{j}
$$

converges absolutely and defines the elements $x_{1}, x_{2}, \cdots$ that appear in the representation (7.2) of $U^{t}$.

Proof. In (7.6) we have $\sum_{j=1}^{\infty}\left|\left\langle v_{j}, y_{i}^{\prime}\right\rangle\right|\left\|u_{j}\right\| \leqq\left\|y_{i}^{\prime}\right\| \sum_{j=1}^{\infty}\left\|v_{j}\right\|\left\|u_{j}\right\|<+\infty$, by (7.4), from which absolute convergence of the series in (7.6) follows. To verify the representation (7.3) by means of (7.6) we see (refer to (2.10) above) that, for $x^{\prime} \in E^{\prime},\left\langle U^{t} x^{\prime}, y_{i}^{\prime}\right\rangle=\sum_{j=1}^{\infty}\left\langle u_{j}, x^{\prime}\right\rangle\left\langle v_{j}, y_{i}^{\prime}\right\rangle=\left\langle x_{i}, x^{\prime}\right\rangle$. Our conclusion follows from this.

Theorem 7.1. Let $t \in E \otimes_{\gamma} F$ be represented by (7.3) and $U^{t}$ by (7.5) and (7.2). Then, the series in

$$
\sum_{i=1}^{\infty}\left\|x_{i}\right\| y_{i}=z
$$

represents an element $z \in F$, and $\|z\| \leqq \gamma(t)$.

Proof. We first show that $z$ belongs to $F$ by means of an indirect argument. With the help of the expressions (7.6) for the $x_{i}$ 's and with a sequence $x_{1}^{\prime}, x_{2}^{\prime}, \cdots, x_{i}^{\prime} \in F^{\prime},\left\|x_{i}^{\prime}\right\| \leqq 1,\left\langle x_{i}, x_{i}^{\prime}\right\rangle=\left\|x_{i}\right\|$, we write formally

$$
\begin{aligned}
z & =\sum_{j=1}^{\infty} \sum_{i=1}^{\infty}\left\langle v_{j}, y_{i}^{\prime}\right\rangle\left\langle u_{j}, x_{i}^{\prime}\right\rangle y_{i} \\
& =\sum_{j=1}^{\infty} w_{j},
\end{aligned}
$$

where

$$
w_{j}=\sum_{i=1}^{\infty}\left\langle v_{j}, y_{i}^{\prime}\right\rangle\left\langle u_{j}, x_{i}^{\prime}\right\rangle y_{i}
$$

Now since the series in the equation $v_{j}=\sum_{i=1}^{\infty}\left\langle v_{j}, y_{i}^{\prime}\right\rangle y_{i}$ converges unconditionally, it follows from the properties of unconditionally convergent series (see in particular [4, top of p. 59]), that the series in (7.9) converges unconditionally. This is because the series in (7.9) is obtained from the series 
for $v_{j}$, by multiplying each term by $\left\langle u_{j}, x_{i}^{\prime}\right\rangle$, and these numbers are bounded. It now follows from the fact that the norm of $F$ is basic with respect to $\Omega$, that $\left\|w_{j}\right\| \leqq\left\|u_{j}\right\|\left\|v_{j}\right\|$. From this and (7.4) it follows that the series $\sum_{j=1}^{\infty} w_{j}$ converges absolutely. Hence $z \in F$ and $\|z\| \leqq \gamma(t)$. Now the $i$ th "Fourier" coefficient of $z,\left\langle z, y_{i}^{\prime}\right\rangle=\sum_{j=1}^{\infty}\left\langle v_{j}, y_{i}^{\prime}\right\rangle\left\langle u_{j}, x_{i}^{\prime}\right\rangle=\left\langle x_{i}, x_{i}^{\prime}\right\rangle=\left\|x_{i}\right\|$. Consequently $z$ is represented by (7.7). This completes the proof of our theorem.

We now rephrase Theorem 7.1 in the form in which it will be usually applied.

Theorem 7.2. A necessary condition in order for a transformation $U$ : $E^{\prime} \rightarrow F$, represented by (7.2), to be nuclear is that $\sum_{i=1}^{\infty}\left\|x_{i}\right\| y_{i}$ be the "Fourier" expansion of an element $z \in F$.

Theorem 7.2 does not by any means solve completely the problem of characterizing nuclear transformations among compact transformations, even in the case when $F$ has an unconditional Schauder basis. Little is known about this problem despite Grothendieck's simple representation (7.3) for elements of $E \otimes_{\gamma} F$. The only infinite dimensional case that seems to be well understood, thanks to the work of Schatten [15], is that in which both $E$ and $F$ are Hilbert spaces, since then nuclear transformations are trace operators. These are simple easily constructed transformations. Theorem 7.2 actually raises a new set of questions, two of which we discuss in this paper. The first is: Can the theorem be used to construct transformations which are compact but not nuclear in cases where the representation (7.3) does not reveal such transformations? The answer is "yes" and we devote the rest of this section to the question. The second question is: Is the condition proved necessary in Theorem 7.2 also sufficient? The answer is "no." We devote the next section to that problem. Let us turn now to the first question for the special case $E=C(S)$.

We have seen that $C(S) \otimes_{\lambda} F$ is the space of all compact linear transformations $U: C^{\prime}(S) \rightarrow F$ that are continuous with respect to the weak * topology of $C^{\prime}(S)$ and the weak topology of $F$. But $C(S) \otimes_{\lambda} F$ is also $C(S, F)(\S 2)$. If $f \in C(S, F)$ corresponds to $U$ under this identification, then for $\mu \in C^{\prime}(S)$,

$$
U_{\mu}=\int_{S} f(s) d \mu .
$$

The basis of $F$ yields the following expansion for $f$

$$
f(s)=\sum_{i=1}^{\infty} \phi_{i}(s) y_{i}
$$

where $\phi_{i}(s) \in C(S)$ and $s \in S$. Now corresponding to Theorem 7.2 we have

Corollary 7.1. A necessary condition for $f \in C(S, F)$ to belong to $C(S)$ $\otimes_{\gamma} F$ is that the relation 


$$
z=\sum_{i=1}^{\infty}\left\|\phi_{i}\right\|_{\infty} y_{i}
$$

be the "Fourier" expansion of an element $z \in F$.

Let us now consider a special case of interest. Let $E=C(S)$. Also let $R$ be a compact Hausdorff space. We know that we can identify the space $C(S \times R)$ with $C[S, C(R)]$. Let the function $\tilde{f} \in C[S, C(R)]$ correspond to $f \in C(S \times R)$ under this identification. Thus for each $s \in S, \tilde{f}(s)$ is the element of $C(R)$ which has the value $f(s, r)$ at $r \in R$.

Corollary 7.2. Suppose that $R$ is a compact Hausdorff space and that $\nu$ is non-negative regular Borel measure on $R$ so that $L_{2}(\nu)$ is separable. Let $\psi_{1}, \psi_{2}, \cdots$ be an orthonormal basis of $L_{2}(\nu)$ consisting of continuous functions. Then a necessary condition for a function $f \in C(S \times R)$ to belong to $C(S)$ $\otimes_{\gamma} C(R)$ is that the expansion

$$
f(s, r)=\sum_{i=1}^{\infty} \phi_{i}(s) \psi_{i}(r)
$$

$\phi_{i} \in C(S)$ and $(s, r) \in S \times R$, satisfy

$$
\sum_{i=1}^{\infty}\left\|\phi_{i}\right\|_{\infty}^{2}<+\infty
$$

Proof. If $f$ belongs to $C(S) \otimes_{\gamma} C(R)$ it has an expansion

$$
f(s, r)=\sum_{j=1}^{\infty} \theta_{j}(s) \eta_{j}(r),
$$

where $\sum_{j=1}^{\infty}\left\|\theta_{j}\right\|_{\infty}\left\|\eta_{j}\right\|_{\infty}<+\infty$. From this it follows that $\sum_{j=1}^{\infty}\left\|\theta_{i}\right\|_{\infty}\left\|\eta_{i}\right\|_{2}$ $<+\infty$, and that $f \in C(S) \otimes_{\gamma} L_{2}(\nu)$. This and Corollary 7.1 imply (7.14).

The importance of Corollary 7.2 is that it gives information about $C(S)$ $\otimes_{\gamma} C(R)$ even when neither $C(S)$ nor $C(R)$ has an unconditional Schauder basis. In particular, if $S=R=I=[0,1]$, it has been asked by various persons if it is possible to find concrete examples of functions belonging to $C(I \times I)$ but not to $C(I) \otimes_{\gamma} C(I)$. Corollary 7.2 makes it possible to give such examples. Let $I_{1}, I_{2}, \cdots$ be an infinite sequence of mutually disjoint subintervals of $I$, each subinterval having positive length. Let us assume, for simplicity, that $I_{j}$ is the open interval $(1 /(i+1), 1 / i)$. Let $\phi_{i}$ $\in C(I)$ be a function vanishing outside of $I_{i}$, and such that $\sum_{i=1}^{\infty}\left\|\phi_{i}\right\|_{\infty}^{2}$ $=+\infty$, but $\left\|\phi_{i}\right\|_{\infty} \rightarrow 0$. It is possible, in this case, to choose the continuous orthonormal functions $\psi_{1}, \psi_{2}, \cdots$, such that $\left\|\psi_{i}\right\|_{\infty}<k$ for some fixed $k>0$. Then the function $f$ given by (7.13) belongs to $C(I \times I)$ but does not satisfy (7.14). 
8. $\Omega$-nuclear transformations. In this section we turn to the second of the two questions asked towards the end of $\$ 7$, namely, is the necessary condition of Theorem 7.2 also sufficient? To this end we introduce a term describing those transformations that satisfy that condition. Our basic assumptions are still that $\Omega=\left\{y_{i}, y_{i}^{\prime}\right\}$ defines an unconditional Schauder basis for $F$ and that the norm of $F$ is basic with respect to $\Omega$. Let $U: E^{\prime} \rightarrow F$ be a bounded linear transformation which is continuous with respect to the weak* topology of $E^{\prime}$ and the weak topology of $F$. We say that $U$ is an (-nuclear transformation if the representation, for $x^{\prime} \in E^{\prime}$,

$$
U x^{\prime}=\sum_{i=1}^{\infty}\left\langle x_{i}, x^{\prime}\right\rangle y_{i}
$$

discussed in $\$ 7$, has the property that the relation

$$
z_{U}=\sum_{i=1}^{\infty}\left\|x_{i}\right\| y_{i}
$$

is the "Fourier" expansion of an element $z_{U} \in F$. See $\$ 7$ for motivation. If $U$ is an $\Omega$-nuclear transformation let $\omega(U)=\left\|z_{U}\right\| \geqq\|U\|$. A routine argument shows that the set of all $\Omega$-nuclear transformations is a vector subspace of $\mathscr{L}\left(E^{\prime}, F\right)$ and is a Banach space under the norm $\omega$.

If $t \in E \otimes F$, then $U^{t}$ is an $\Omega$-nuclear transformation by Theorem 7.2. We again let $\omega(t)=\omega\left(U^{t}\right)$. We see immediately that $\gamma \geqq \omega \geqq \lambda$. Consequently $\omega$ is a cross norm and defines a complete tensor product $E \otimes_{\omega} F$. If $t \in E \otimes_{\omega} F$, then clearly $U^{t}$ is an $\Omega$-nuclear transformation. Conversely every $\Omega$-nuclear transformation $U: E^{\prime} \rightarrow F$ is of the form $U^{t}, t \in E \otimes_{\omega} F$, for if $U$ is represented by (8.1), satisfying (8.2), let $U_{n}: E^{\prime} \rightarrow F$ be defined, for $x^{\prime} \in E$, by

$$
U_{n} x^{\prime}=\sum_{i=1}^{n}\left\langle x_{i}, x^{\prime}\right\rangle y_{i}
$$

Then $U_{n}=U^{t_{n}}$, for $t_{n}=\sum_{i=1}^{n} x_{i} \otimes y_{i}$ and $\omega\left(U-U_{n}\right)=\left\|\left(\sum_{i=n+1}^{\infty}\left\|x_{i}\right\| y_{i}\right)\right\| \rightarrow 0$. Hence $E \otimes_{\omega} F$ can be isometrically identified with the space of all $\Omega$-nuclear transformations. In particular, every $\Omega$-nuclear transformation is compact.

We say that $F$ is $\Omega$-nuclear if, for every Banach space $E$, every $\Omega$-nuclear transformation $U: E^{\prime} \rightarrow F$ is nuclear, that is, of the form $U^{t}, t \in E \otimes_{\gamma} F$.

Two examples may clarify the situation. If both $E$ and $F$ are the same separable Hilbert space $H, \Omega$ defines an orthonormal basis $y_{1}, y_{2}, \cdots$ of $H$, and $U: H \rightarrow H$, under a suitable identification of $H$ and $H^{\prime}$, we can easily see that in the representation (8.1), we have $x_{i}=U^{*} y_{i}$, for every $i$ where $U^{*}$ is the adjoint operator of $U$. Hence (8.2) becomes

$$
\sum_{i=1}^{\infty}\left\|U^{*} y_{i}\right\|^{2}<+\infty
$$


We easily recognize (8.4) as the definition of the Schmidt class of Schatten $[15$, Definition 5.1, p. 73]. Hence for Hilbert spaces our notion of $\Omega$-nuclear transformations reduces to that of Schmidt operators, and $\omega(U)$ reduces to the Schmidt norm. Thus $H \otimes_{\omega} H$ is the Schmidt class. Since it is well known that not every Schmidt operator is nuclear (of the trace class, in the terminology of Schatten) we see that Hilbert spaces are not $\Omega$-nuclear if $\Omega$ defines an orthonormal basis.

Our next example concerns $F=l_{1}$ and $\Omega$ the standard biorthogonal system. $E$ is an arbitrary Banach space. In this case (8.2) becomes

$$
\sum_{i=1}^{\infty}\left\|x_{i}\right\|<+\infty \text {. }
$$

Consequently, if $U$ is an $\Omega$-nuclear transformation, then $U=U^{t}$, where $t=\sum_{i=1}^{\infty} x_{i} \otimes y_{i}, \quad \sum_{i=1}^{\infty}\left\|x_{i}\right\|\left\|y_{i}\right\|=\sum_{i=1}^{\infty}\left\|x_{i}\right\|<+\infty$. Hence $t \in E \otimes_{\gamma} F$, and $U$ is nuclear. Therefore $l_{1}$ is $\Omega$-nuclear. In this section we show that $l_{1}$ is essentially the only $\Omega$-nuclear space.

We can now answer the question that motivated this section. Theorem 7.2 states, in our new terminology, that a necessary condition for a bounded linear transformation $U: E^{\prime} \rightarrow F$, which is continuous with respect to the weak $^{*}$ topology of $E^{\prime}$ and the weak topology of $F$, to be nuclear is that it be $\Omega$-nuclear. It is sufficient for all such transformations into $F$ if and only if $F$ is $\Omega$-nuclear, hence not sufficient in general, since not all spaces $F$ are $\Omega$-nuclear with respect to every $\Omega$ defining an unconditional Schauder basis for $F$. We have shown separable Hilbert space to be an exception.

The space $\mathscr{L}\left(E, F^{\prime}\right)$ has a rather simple structure if $F$ is $\Omega$-nuclear. The next lemma gives a description of $\mathscr{L}\left(E, F^{\prime}\right)$. We now lay the grounds for its proof. As in $\S 7$, we have $E \otimes_{\tau} F=E \otimes_{\gamma} F$ isometrically. Under our present assumption that $F$ is $\Omega$-nuclear, we also have

$$
E \otimes_{\omega} F=E \otimes_{\tau} F=E \otimes_{\gamma} F,
$$

where the first equality for the moment represents merely an algebraic isomorphism. However, from $\gamma \geqq \omega$ and the fact that all spaces involved are complete relative to their respective norms, it follows with the help of the open mapping theorem that $\omega$ and $\gamma$ are equivalent norms. It then follows that there exists a positive number $\Delta$ so that, for every $t \in E \otimes_{\omega} F$ we have

$$
\gamma(t) \leqq \Delta \omega(t) .
$$

Let now $T: E \rightarrow F^{\prime} . T$ has a representation similar to the representation (8.1) for $U: E^{\prime} \rightarrow F$. There exist elements $x_{1}^{\prime}, x_{2}^{\prime}, \cdots \in E^{\prime}$ so that $\left({ }^{3}\right)$ for every $x \in E$,

$\left({ }^{3}\right)$ The star represents, as in $\$ 6$, the weak* convergence of the series in question. 


$$
T x=^{*} \sum_{i=1}^{\infty}\left\langle x, x_{i}^{\prime}\right\rangle y_{i}^{\prime}
$$

Lemma 8.1. If $F$ is $\Omega$-nuclear then for every Banach space $E$ and every bounded linear transformation $T: E \rightarrow F^{\prime}$, the representation (8.8) of $T$ has the property that the equation

$$
\boldsymbol{z}^{\prime}=^{*} \sum_{i=1}^{\infty}\left\|x_{i}^{\prime}\right\| y_{i}^{\prime}
$$

defines an element $\boldsymbol{z}^{\prime} \in F^{\prime}$.

Proof. Suppose that $F$ is $\Omega$-nuclear, and let $T: E \rightarrow F^{\prime}$ be a bounded linear transformation represented by (8.8). Let $y \in F$. Then $y$ has the expansion

$$
y=\sum_{i=1}^{\infty}\left\langle y, y_{i}^{\prime}\right\rangle y_{i}
$$

Let us also consider a sequence $\mathscr{S}: x_{1}, x_{2}, \cdots$, with $x_{i} \in E,\left\|x_{i}\right\| \leqq 1$. With the pair $y, \mathscr{S}$ we associate a bounded linear transformation $U_{y, S}: E^{\prime} \rightarrow F$ defined, for every $x^{\prime} \in E^{\prime}$, by

$$
U_{y, \zeta} x^{\prime}=\sum_{i=1}^{\infty}\left\langle x_{i}, x^{\prime}\right\rangle\left\langle y, y_{i}^{\prime}\right\rangle y_{i}
$$

From the unconditional convergence of the series in (8.10) and the fact that the sequence $\mathscr{S}$ is bounded it follows that $\sum_{i=1}^{\infty}\left\|x_{i}\right\|\left|\left\langle y, y_{i}^{\prime}\right\rangle\right| y_{i}$ converges unconditionally. Hence $U_{y, \zeta}$ is $\Omega$-nuclear. Then from our hypothesis we conclude that $U_{y, \mathscr{S}}$ is nuclear and there exists $t_{y_{r}} \mathscr{E} \in E \otimes_{\gamma} F$ so that $U_{y, S}=U^{t}, \mathscr{Y}$.

Let us return now to $T$. From the duality theorem for $E \otimes_{\gamma} F$ (see \$2), there exists $t_{T}^{\prime} \in\left(E \otimes_{\gamma} F\right)^{\prime}$, for which the following computations are justified.

$$
\begin{aligned}
\left\langle t_{y, \mathscr{S}}, t_{T}^{\prime}\right\rangle & =\sum_{i=1}^{\infty}\left\langle x_{i}, x_{i}^{\prime}\right\rangle\left\langle y, y_{i}^{\prime}\right\rangle \leqq\|T\| \gamma\left(t_{y, \mathscr{S}}\right) \\
& \leqq \Delta\|T\| \omega\left(t_{y, \mathscr{S}}\right) \leqq \Delta\|T\|\|y\| .
\end{aligned}
$$

Since $\mathscr{S}$ is arbitrary except for the restriction that $\left\|x_{i}\right\| \leqq 1$, we see that the sign of each $\left\langle x_{i}, x_{i}^{\prime}\right\rangle$ may be chosen arbitrarily. Hence, from (8.12) we have

$$
\sum_{i=1}^{\infty}\left|\left\langle x_{i}, x_{i}^{\prime}\right\rangle\right|\left|\left\langle y, y_{i}^{\prime}\right\rangle\right| \leqq \Delta\|T\|\|y\|
$$

Again, because of our free choice of $\mathscr{S}$ we deduce that 


$$
\sum_{i=1}^{\infty}\left\|x_{i}^{\prime}\right\|\left|\left\langle y, y_{i}^{\prime}\right\rangle\right| \leqq\|T\|\|y\| .
$$

Hence (8.9) defines an element $z^{\prime} \in F^{\prime}$.

THEOREM 8.1. The space $F$ is $\Omega$-nuclear if and only if $\Omega$ defines an absolute Schauder basis for $F$.

Proof. If $\Omega$ defines an absolute Schauder basis for $F$, then $F$ is essentially $l_{1}$, and $\Omega$ corresponds to the standard biorthogonal system. It follows from our discussion of $l_{1}$ above that $F$ is $\Omega$-nuclear.

Conversely, suppose that $F$ is $\Omega$-nuclear. The identity mapping $J: F^{\prime} \rightarrow F^{\prime}$ has a representation

$$
J x^{\prime}=\sum_{i=1}^{\infty}\left\langle y_{i}, x^{\prime}\right\rangle y_{i}^{\prime},
$$

corresponding to (8.8). Consequently, by Lemma 8.1, $z^{\prime}={ }^{*} \sum_{i=1}^{\infty} y_{i}^{\prime} \in F^{\prime}$. Now, for any $y=\sum_{i=1}^{\infty}\left\langle y, y_{i}^{\prime}\right\rangle y_{i} \in F, \sum_{i=1}^{\infty}\left|\left\langle y, y_{i}^{\prime}\right\rangle\right| y_{i}$ also converges to an element $\tilde{z} \in F$. Now $\left\langle\tilde{z}, z^{\prime}\right\rangle=\sum_{i=1}^{\infty}\left|\left\langle y, y_{i}^{\prime}\right\rangle\right|<+\infty$. Hence $\Omega$ defines an absolute Schauder basis.

We have thus characterized $\Omega$-nuclear spaces. The next corollary gives an application of Lemma 8.1 to obtain a concrete representation of the general bounded linear transformation of $E$ into $l_{\infty}$. No doubt this is a known result, although we know of no specific reference.

Corollary 8.1. There is an isometric isomorphism of $\mathscr{L}\left(E, l_{\infty}\right)$ onto the space $l_{\infty}\left(E^{\prime}\right)$ of all bounded sequences $\mathscr{S}=\left\{x_{1}^{\prime}, x_{2}^{\prime}, \ldots\right\}, x_{i}^{\prime} \in E^{\prime}$, where $\|\mathscr{S}\|_{\infty}$ is defined as $\sup _{i}\left\|x_{i}^{\prime}\right\|$, in such a way that if $\mathscr{S}^{T} \in l_{\infty}\left(E^{\prime}\right)$ corresponds to $T \in \mathscr{L}\left(E, l_{\infty}\right)$ under this isomorphism, then, for every $x \in E$,

$$
T x=\left(\left\langle x, x_{1}^{\prime}\right\rangle,\left\langle x, x_{2}^{\prime}\right\rangle, \cdots\right),
$$

where $\left\{x_{1}^{\prime}, x_{2}^{\prime}, \cdots\right\}=\mathscr{S}^{T}$.

Proof. Since $l_{1}$ is an $\Omega$-nuclear space with respect to the usual $\Omega$, any bounded linear transformation $T: E \rightarrow l_{1}^{\prime}=l_{\infty}$ has a representation (8.8) satisfying the condition embodied in (8.9). In this case the fact that $z^{\prime}$ (in Lemma 8.1) belongs to $l_{\infty}$, means that the sequence $\mathscr{S}_{T}=\left\{x_{1}^{\prime}, x_{2}^{\prime}, \ldots\right\}$ is bounded. Equation (8.8) becomes (8.16). That in this case $\left\|\mathscr{S}_{T}\right\|=\|T\|$ follows quickly from (8.16), hence the correspondence $T \rightarrow \mathscr{S}^{T}$ is isometric. It is clearly onto. This completes the proof.

From the general relation $L_{1}(\mu, E)=L_{1}(\mu) \otimes_{\gamma} E$ (see §2), it follows that the space $l_{1}(E)$ of absolutely summable $E$-sequences, with its natural norm, is $l_{1} \otimes_{\gamma} E$. These considerations and Corollary 8.1 yield immediately the following corollary. 
COROLlaRY 8.2. There is an isometric isomorphism expressed by the following equation:

$$
l_{1}^{\prime}(E)=l_{\infty}\left(E^{\prime}\right)
$$

in such a way that if $\mathscr{S}$ represents the sequence $\left\{x_{1}, x_{2}, \cdots\right\} \in l_{1}(E)$ and $\mathscr{S}^{\prime}$ the sequence $\left\{x_{1}^{\prime}, x_{2}^{\prime}, \cdots\right\} \in l_{\infty}\left(E^{\prime}\right)$ then

$$
\left\langle\mathscr{S}, \mathscr{S}^{\prime}\right\rangle=\sum_{i=1}^{\infty}\left\langle x_{i}, x_{i}^{\prime}\right\rangle
$$

\section{BIBLIOGRAPHY}

1. R. G. Bartle, A general bilinear vector integral, Studia Math. 15 (1956), 337-352.

2. R. G. Bartle, N. Dunford and J. Schwartz, Weak compactness and vector measures, Canad. J. Math. 7 (1955), 289-305.

3. N. Bourbaki, Eléments de mathématiques. VI. Intégrations, Actualités Sci. Ind. No. 1281, Hermann, Paris, 1952.

4. M. M. Day, Normed linear spaces, Ergebnisse der Mathematik und ihrer Grenzgebiete, Heft 21, Springer, Berlin, 1958.

5. N. Dinculeanu, Mesures vectorielles et opérations linéaires, C. R. Acad. Sci. Paris 246 (1958), 2328-2331.

6. N. Dinculeanu and C. Foias, Mesures vectorielles et opérations linéaires sur $L_{E}^{p}, \mathrm{C} . \mathrm{R}$. Acad. Sci. Paris 248 (1959), 1759-1762.

7. N. Dunford and J. Schwartz, Linear operators, Vol. I, Interscience, New York, 1958.

8. B. R. Gelbaum and J. Gil de Lamadrid, Bases of tensor products of Banach spaces, Pacific J. Math. 11 (1961), 1281-1286.

9. J. Gil de Lamadrid, On finite dimensional approximations of mappings in Banach spaces, Proc. Amer. Math. Soc. 10 (1962), 163-168.

10. A. Grothendieck, Produits tensoriels topologiques et espaces nucléaires, Mem. Amer. Math. Soc. No. 16 (1955), 140 pp.

11. __ La théorie de Fredholm, Bull. Soc. Math. France 84 (1956), 319-384.

12. A. Ionescu Tulcea and $\mathrm{C}$. Ionescu Tulcea, On the decomposition and integral representations of continuous linear operators, Ann. Mat. Pura Appl. 53 (1961), 81-88.

13. On the lifting property. II. Representation of linear operators on the spaces $L_{E}^{r}, 1 \leqq r<\infty$, J. Math. Mech. 7 (1962), 773-795.

14. R. S. Phillips, On linear transformations, Trans. Amer. Math. Soc. 48 (1938), 516-541.

15. R. Schatten, $A$ theory of cross-spaces, Annals of Mathematics Studies No. 26, Princeton Univ. Press, Princeton, N. J., 1950.

16. J. Schauder, Eine Eigenschaft des Haarschen Orthogonal systems, Math. Z. 28 (1928), 317-320.

17. I. Singer, Linear functionals on spaces of continuous mappings of a compact Hausdorff space into a Banach space, Rev. Math. Pures Appl. 2 (1957), 309-315. (Russian)

\footnotetext{
UNIVERSITY OF MinNESOTA,

MinNeApolis, MinNesota

Yale University,

New Haven, Connecticut
} 